\title{
Environment Manipulation Planner for Humanoid Robots Using Task Graph That Generates Action Sequence
}

\author{
Kei Okada Atsushi Haneda Hiroyuki Nakai Masayuki Inaba Hirochika Inoue \\ Graduate School of Information Science and Technology, The University of Tokyo, Tokyo, JAPAN \\ Email: k-okada@jsk.t.u-tokyo.ac.jp
}

\begin{abstract}
In this paper, we describe a planner for a humanoid robot that is capable of finding a path in an environment with movable objects, whereas previous motion planner only deal with an environment with fixed objects. We address an environment manipulation problem for a humanoid robot that finds a walking path from the given start location to the goal location while displacing obstructing objects on the walking path. This problem requires more complex configuration space than previous researches using a mobile robot especially in a manipulation phase, since a humanoid robot has many degree of freedoms in its arm than a forklift type robot. Our approach is to build environment manipulation task graph that decompose the given task into subtasks which are solved using navigation path planner or whole body motion planner. We also propose a standing location search and a displacing obstacle location search for connecting subtasks. Efficient method to solve manipulation planning that rely on whole body inverse kinematics and motion planning technology is also shown. Finally, we show experimental results in an environment with movable objects such as chairs and trash boxes. The planner finds an action sequence consists of walking paths and manipulating obstructing objects to walk from the start position to the goal position.
\end{abstract}

\section{Introduction}

Recently as a highly stable walking control technology progresses, much attention to high level control of a humanoid robot is paid. Motion planning method of a humanoid robot is the one of the demanded high level behavior control technique in these days [1].

Previous researches on a humanoid robot motion planner ware mainly classified as a navigation planner that finds collision free walking path from the start location to the goal location or as an arm/body motion planner that calculates a collision free trajectory from the start posture to the goal posture.

In this paper, we propose new planning problem for a humanoid robot that a robot have to find a walking path from the given initial location to the goal location while displacing obstructing objects, which we call a environment manipulation task(Fig.1 ).

Motion planning is one of the intensively researched area in a robotics field, however these researches use mobile robots [2]. Manipulation planning for humanoid robots require more complex configuration space than that of mobile robots, since a humanoid robot has many degree of freedoms than a mobile robot or a forklift type robot.

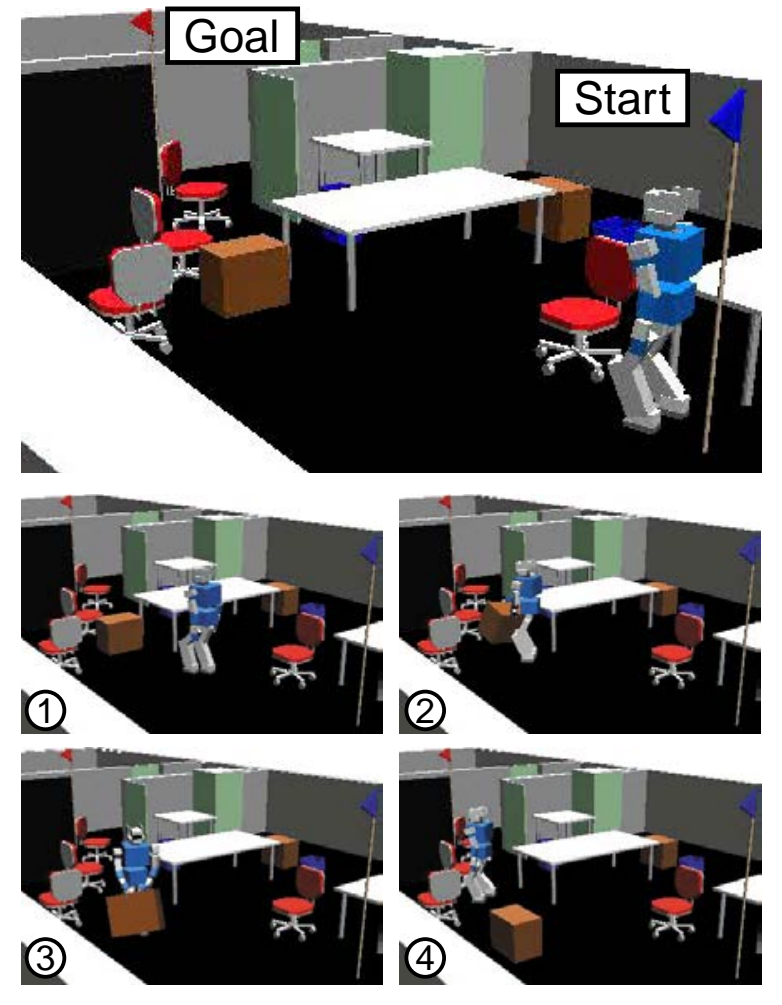

Fig. 1. Environment manipulation planning problem for a humanoid robot

Therefore, in this paper, we propose a practical planner for a humanoid robot that is capable of finding a walking path from the given initial location to the goal location while displacing obstructing objects, which we call a environment manipulation task.

Our approach is to build environment manipulation task graph that decomposes the given task into subtasks which are represented as navigation motion planner and whole body motion planner. In order to connect each motion planner continuously, we introduce new problems for humanoid robot that we call standing location search and displacing obstacle location search.

\section{Related Work}

\section{A. Navigation Planning}

Navigation planning tasks are defined as obtaining a collision free path of a robot from the initial location to 
the goal location, by searching a two dimensional configuration space that represents position of a robot, or a three dimensional configuration space that represents position and orientation of a robot.

For humanoid robots, conventional navigation planning methods are able to applied by using a bounding volume of a robot such as a box or a cylinder, and controls a humanoid robot along with a computed path $[3]-[7]$.

Several researchers have been tackled a footprint planner [8]-[10] that finds a sequence of footprints from the initial location to the goal location while avoiding obstacles by stepping over or taking a devious route.

\section{B. Arm/Body Posture Planning}

Arm/body posture planning tasks are defined as computing an arm or a whole body motion trajectory from the initial posture to the goal posture while avoiding obstacles.

Due to the high degree of freedoms of a humanoid robot or its arm, arm/body planner requires highdimensional configuration spaces. Therefore the randomized algorithms are commonly used instead of the complete algorithm for posture planning of a humanoid robot [11], [12].

\section{Manipulation Planning}

Manipulation planning tasks are defined as moving a target object to its new location by a robot(s) [13]. A solution of manipulation planning problem is a sequence of transit paths (a robot moves while holding an object) and transfer paths (a robot moves alone) separated by grasp and ungrasp operations. This planner realizes interesting and intelligent behavior of a robot in an environment with movable objects whereas conventional motion planner only concerns environment with static obstacles.

There are many researches on manipulation planning so far. For example, Gravot et al. have proposed one of the sophisticated method that deal with multiple robots with movable objects by using a set of multiple probabilistic roadmaps [14]. Although they used simple forklift type mobile robots that can lift it's palette, the dimensions for the whole configuration space becomes so large. Thus, they decompose the problem into several roadmaps that is transfer, transit, grasp and placement, then apply probabilistic roadmap method for finding solution in each roadmap and elementary kinematic graphs for connecting them.

\section{Contributions of This Paper}

In this paper, we propose a practical planner for a humanoid robot that is capable of finding a walking path from the given initial location to the goal location while displacing obstructing objects, which we call a environment manipulation task. This problem requires more complex configuration space than previous manipulation planning research using mobile robots,

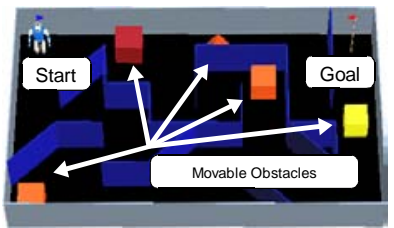

Environment Manipulation Task

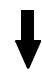

1

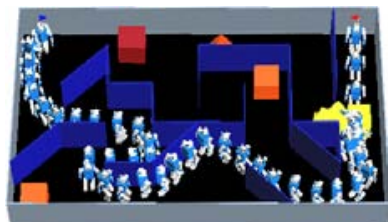

Environment Manipulation Motions

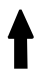

4

Environment Manipulation Planner

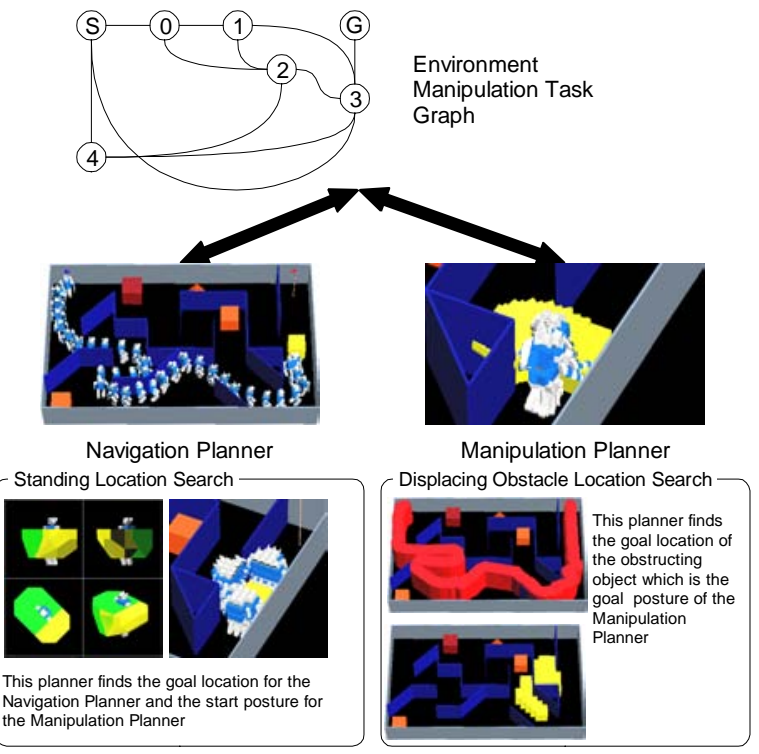

Fig. 2. An Overview of Environment Manipulation Planning for a Humanoid Robot

especially manipulation phase, since a humanoid robot has many degree of freedoms in its arm than a forklift type robot. Our approach is to build environment manipulation task graph that decomposes the given task into subtasks which are represented as navigation motion planner and whole body motion planner.

In order to connect each motion planner continuously, we introduce new problems for humanoid robot that we call standing location search and displacing obstacle location search. The standing location search finds a position to stand for manipulating a object. The displacing obstacle location search finds a goal location of a obstructing object to ensure walking through path. We also introduce whole body manipulation planner that rely on whole body inverse kinematics and motion planning technology.

\section{Environment Manipulation Planner for Humanoid Robot}

We propose a environment manipulation planner of a humanoid robot which a robot tries to find a walking path from the initial location to the goal location while displacing obstructing objects on the trajectory. Our environment manipulation planner is composed of environment manipulation task planner, navigation motion planner, manipulation motion planner as shown 


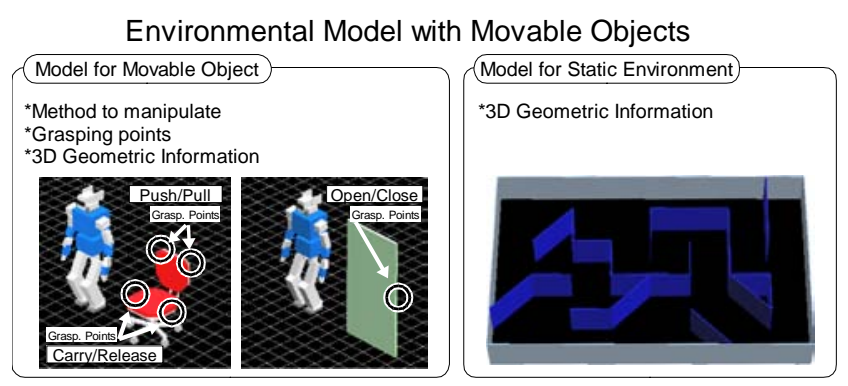

Fig. 3. Examples of Environment Model with Movable Objects

in Fig.2 .

Environment Manipulation Task Planner

This planner build environment manipulation task graph for decomposing the given task to subtasks which are navigation and manipulation motion planner. Standard graph search algorithms find action sequence that which obstacles to be removed and which route to walk from the start location to the goal location. This graph is automatically build using environment model with movable objects.

Navigation motion planner

A navigation motion planner finds a collision free walking path of a humanoid robot between the start location to the obstacle to be removed, between obstacles or the obstacle to the goal location. Goal locations for each navigation motion planner but the last one, are determined by standing location search method which finds a position to stand for manipulating the obstacle to be removed.

Manipulation motion planner

A manipulation motion planner computes a collision free whole body trajectory for displacing obstructing objects on the walking path, by taking the given initial posture of a humanoid robot and the goal location of the object. The goal location of the obstructing object is calculated using displacing obstacle location search method which finds a goal location of the object in order to ensure collision free walking path.

IV. Environmental Model with Movable Objects

Our environmental model with movable objects contains 3D geometric model for fixed objects and 3D geometric model, grasping points and a method to manipulate a object for movable objects as shown in Fig.3 . This information is used for our task and motion planner.

\section{Environment Manipulation Task Planner}

This planner build search graph for decomposing the given task to subtasks which are navigation and manipulation motion planner. This search graph, which

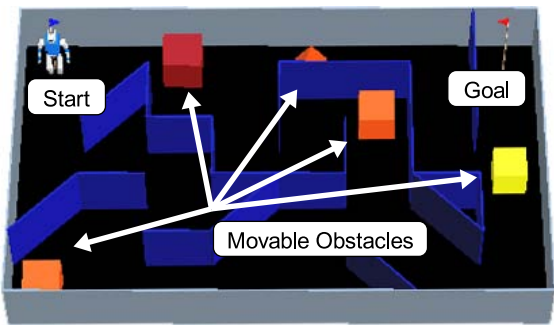

(a)

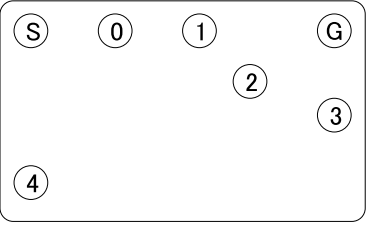

(b)

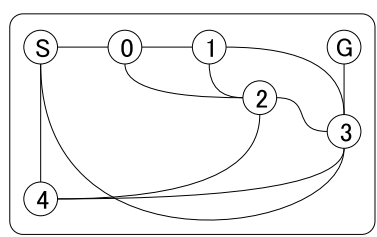

(c)
Fig. 4. Environment manipulation task planner: (a) Environmental model with the start and the goal location, (b) Generated nodes for environment manipulation task graph, (c) Environment manipulation task graph
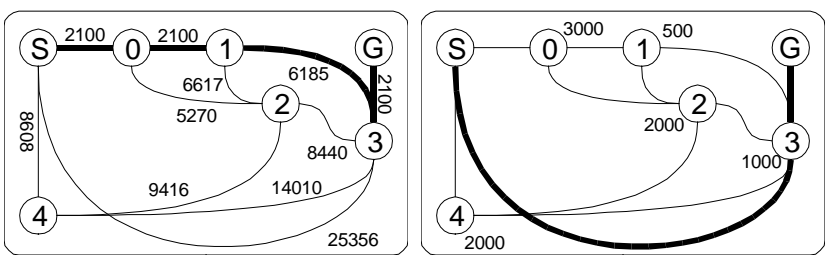

Fig. 5. Result of environment manipulation task planner. The left figure shows a minimum distance solution and the right figure shows a minimum workload solution.

we call environment manipulation task graph, consists of nodes which correspond to movable objects and arcs which represent an existence of collision free walking path between each movable object. Fig.4 illustrates the procedure to build the search graph.

(a) User gives the starting location and the goal location in the environmental model with movable objects.

(b) Planner generates nodes which correspond to movable objects.

(c) Planner generates arcs between each node if there exists collision free walking path between nodes by invoking navigation planner.

\section{A. Solution of Environment Manipulation Task Graph}

Searching the environment manipulation search graph finds a solution of given task that which object a robot need to displace and which route to walk.

Our planner is capable of select a strategy for finding a solution, for example when we set a length of each nodes as a cost functions, it find a minimum distance solution from the start location to the goal position. On the other hand, when we use a workload as a cost that is weights of movable objects and procedures of 

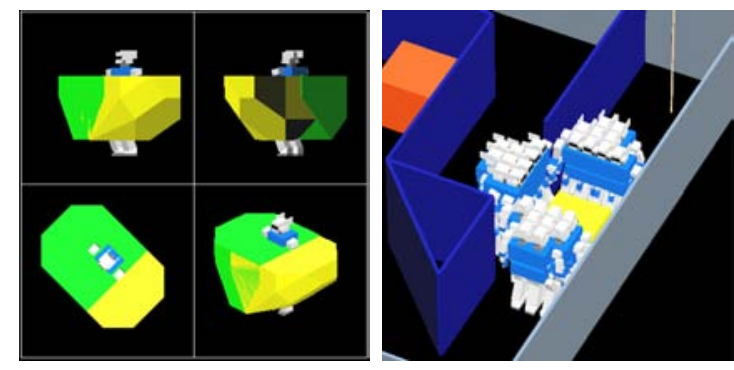

Fig. 6. Left) Reachable area of a humanoid robot (green:rightarm,yellow:left-arm), Right) Result of standing location candidates

manipulation, it finds a solution with a minimum total workload.

Fig.5 shows these examples. The left figure shows a minimum distance solution and the right figure shows a minimum workload solution.

\section{Navigation Motion Planner}

This planner finds a collision free walking path of a humanoid robot between the start location to the displacing object, between displacing objects or the displacing object to the goal location. Goal location for each navigation planner are determined by standing location search method which finds a position of a humanoid robot to manipulate the displacing object.

\section{A. Standing Location Search}

Standing location search for a humanoid robot relies on a reachable area calculation and a standing location candidates.

The reachable area is a set of points where a humanoid robot is able to reach by it's hand while satisfying stability. In order to examine if a humanoid robot is able to reach each point, we adopt pinand-drag interface for whole body inverse kinematics calculation [15]. When this inverse kinematics method find a solution, the point is considered as within reachable area. Fig.6 (Left) shows results of reachable area calculation.

Standing location candidates are calculated using reachable area and grasping points. Given a target object to manipulate and a set of standing location points of a humanoid robot around the object, if grasping points is within reachable area, this standing location is considered as standing location candidates. Fig.6 (Right) shows standing location candidates.

Among standing location candidates, standing location that a robot does not collide with an environment and closest orientation along with the path is selected as the goal location for navigation motion planner.

\section{B. Collision Free Path Planner}

This planner finds collision free walking path from the given starting point to the standing location calculated by standing location search. We use C-space

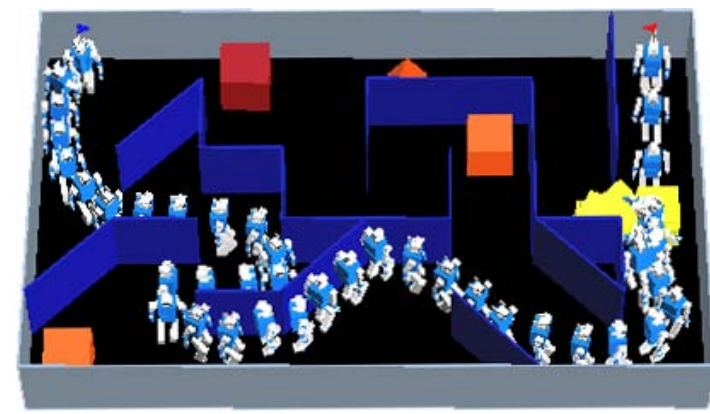

Fig. 7. Result of navigation motion planning

of $x, y, \theta$ that represents position and orientation of a humanoid robot. Result of collision free path from the starting location to the goal location where is calculated by standing location search using the given manipulatable object, is shown in Fig.7 .

\section{Manipulation Motion Planner}

This planner takes an displacing object and an environment, then it finds generate whole body posture sequence of a humanoid robot for moving the target object in order to ensure a collision free navigation path to the destination. The procedure of this planner is, first it finds goal location of a movable object which does not collide with free walking path, then it calculates whole body posture sequence for displacing the target object from current position to the goal position.

\section{A. Displacing Obstacle Location Search}

In order to determine goal location a movable object, first it determine collision free walking path from the start location to the goal location as shown in Fig.9 (Left). Then it calculates goal location candidates of obstructing object using the range of movable object that information is described in environment model with movable objects. Among these location candidates, locations which overlap with collision free walking path. The rest are the goal location candidates of movable objects as shown in Fig.9 (Right).

\section{B. Whole Body Motion Planning of Humanoid Robot}

This planner calculates whole body posture sequence of a humanoid robot in order to manipulate a movable object from the current location to the goal location. The goal location is selected from the goal location candidates under the criteria that the closest goal location of the candidates from the current position.

Whole body posture sequence of a humanoid robot from the current posture to the goal posture is calculated using whole body collision-free motion planning method [16].

The result of environment manipulation planner which manipulates a movable object in order to ensure collision free walking path, is shown in Fig.8 . 

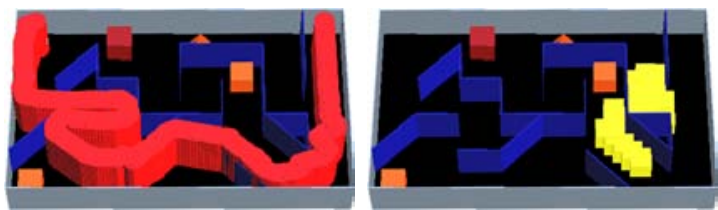

Fig. 8. (Left) Collision free path from the start location to the goal location. (Right) The goal location candidates of the displacing object.

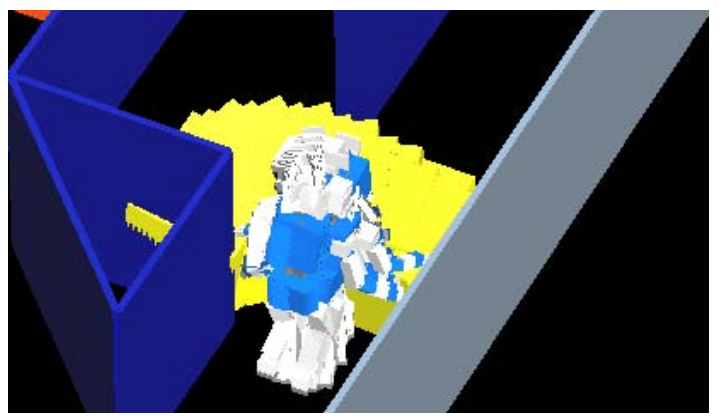

Fig. 9. The result of whole body motion sequence of a humanoid robot

\section{Experimental Results of Environment Manipulation Planner}

In this section, we demonstrate the example of environment manipulation planner. In this experiment, we build an environmental model of our laboratory, movable object model such as chairs, trash boxes and tables, and a robot model that has the same kinematics and joint limit information of the HRP2 humanoid robot [17] which displayed in Fig.10 . Fig.11 shows environment manipulation task graph.

This task graph is used to find environment manipulation task sequence that is composed of walking path between the start location to the obstacle to be removed, between obstacles or the obstacle to the goal location, and whole body manipulation posture sequence for displacing obstructing objects in order to ensure collision free walking path from the start location to the goal location.

Fig.12 shows the result of task planning using shortest path criteria. The humanoid robot remove the brown box(5), the red chair(2) and the red chair(3) in order to walk through from the start location to the goal location.

Fig.13 also shows the result of task planner, but it uses minimum workload criteria. The robot removes only two obstructing objects that is the blue trash box (7) and the red chair(3).

\section{Conclusions}

We describe a planner for a humanoid robot that is capable of finding a path in an environment with movable objects while displacing obstructing objects by whole body manipulation in order to ensure collisionfree walking path, which we call an environment manip-

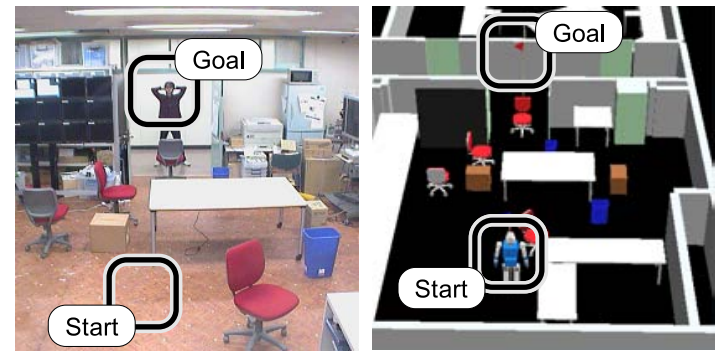

Fig. 10. An environment and movable objects model

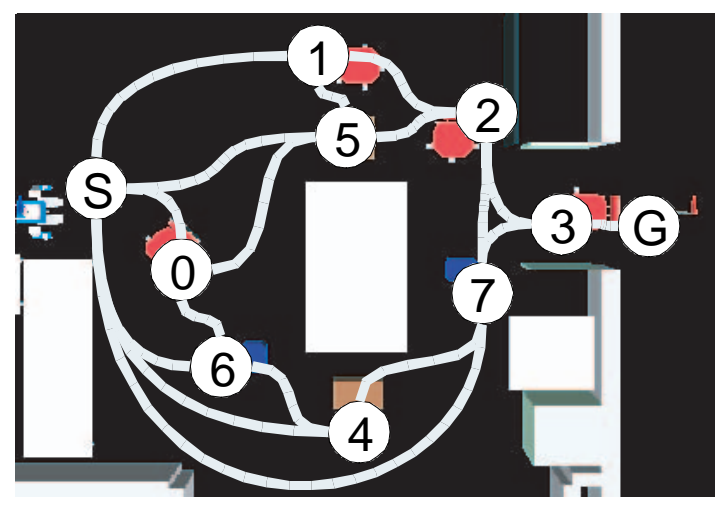

Fig. 11. An environment manipulation task graph

ulation task. Though this task requires more complex configuration space than conventional manipulation task for mobile robots, Our approach to build environment manipulation task graph to decompose the given task into navigation and manipulation subtasks does not suffer from high complexity of the task. We also propose standing location search and displacing obstacle location search for connecting subtasks. Efficient method to solve manipulation planning that rely on whole body inverse kinematics and motion planning technology is also shown. An experimental results of this planner in an environment with movable objects such as chairs and trash boxes that it finds an action sequence consists of walking paths and manipulating obstructing objects are shown.

\section{References}

[1] J.J. Kuffner and K. Nishiwaki and S. Kagami and M. Inaba and H. Inoue. Motion planning for humanoid robots. In In Proceedings of 11th International Symposyum on Robotics Research (ISRR'03), page 20, 2003.

[2] N. Nilsson. Shakey the robot. A.i. center technical note 323, SRI International, 1984

[3] Z. Shiller, K. Yamane, and Y. Nakamura. Planning motion patterns of human figures using a multi-layered grid and the dynamics filter. In In Proceedings of the IEEE International Conference on Robotics and Automation (ICRA'01), pages $1-8,2001$

[4] Y. Sakagami, R. Watanabe, C. Aoyama, S. Matsunaga, N. Higaki, and K. Fujimura. The intelligent ASIMO: System overview and integration. In Proceedings of the 2002 IEEE/RSJ International Conference on Intelligent Robots and Systems (IROS'02), pages 2478-2483, 2002.

[5] K. Sabe, T. Ohashi, K. Kawamoto, J.S. Gutmann, M. Fukuchi, and T. Yoshigahara. Obstacle avoidance and 


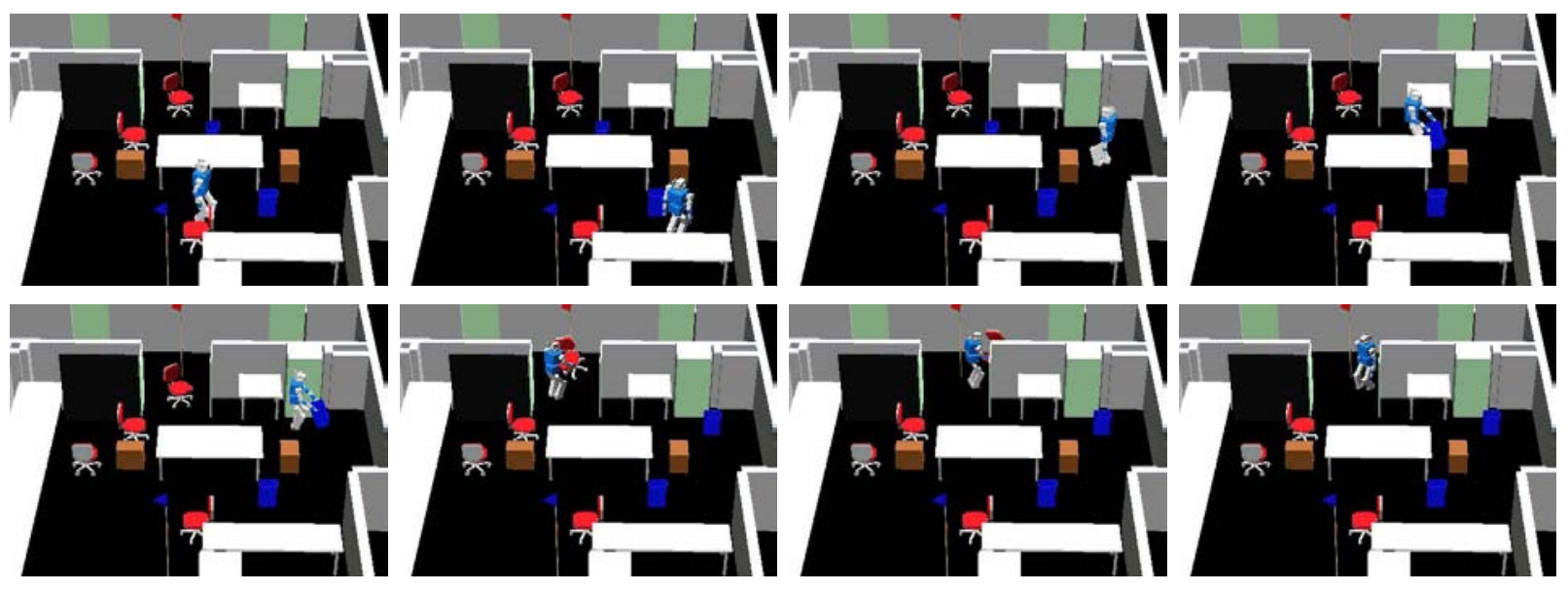

Fig. 12. The result of searching task graph under shortest distance criteria

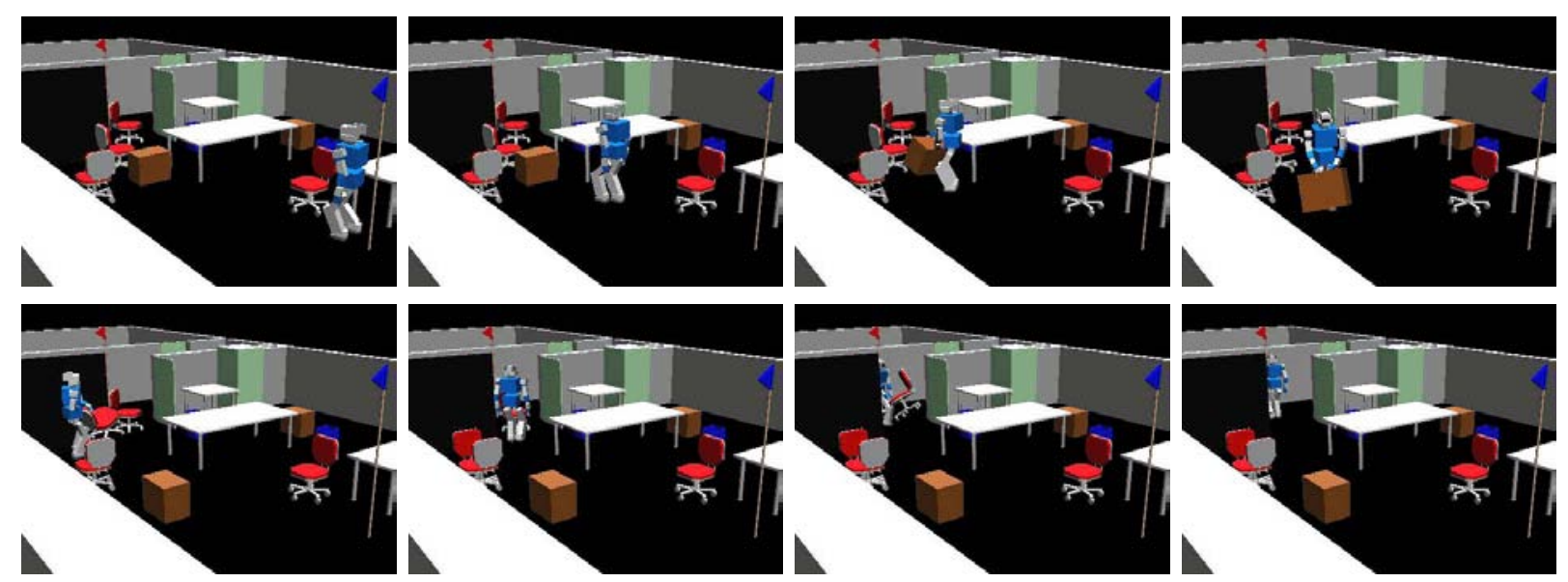

Fig. 13. The result of searching task graph under minimum workload criteria

path planning for humanoid robot using stereo vision. In Proceedings of National Symposium on Sensing via Image Information (SSII'02), 2002 (In Japanese).

[6] K. Nishiwaki, S.Kagami, J.J. Kuffner, K. Okada, Y. Kuniyoshi, M. Inaba, and H. Inoue. Online Humanoid Walking Control and 3D Vision-based Locomotion, volume 5 of Springer Tracts in Advanced Robotics, pages 85-94. Springer, 2003.

[7] K. Okada, M. Inaba, and H. Inoue. Integration of realtime binocular stereo vision and whole body information for dynamic walking navigation of humanoid robot. In Proceedings of International Conference on Multisensor Fusion and Integration for Intelligent Systems (MFI'03), pages 131-136, 2003.

[8] J.J. Kuffner, K. Nishiwaki, S. Kagami, M. Inaba, and H. Inoue. Footstep planning among obstacles for biped robots. In Proceedings of the IEEE/RSJ International Conference on Intelligent Robots and Systems (IROS'01), pages 500-505, 2001.

[9] O. Lorch, A. Albert, J. Denk, M. Gerecke, R. Cupec, J.F. Seara, W. Gerth, and G. Schmidt. Experiments in visionguided biped walking. In Proceedings of the IEEE/RSJ International Conference on Intelligent Robots and Systems (IROS'02), pages 2484-2490, 2002.

[10] J. Chestnutt and J.J. Kuffner and K. Nishiwaki and S. Kagami. Planning biped navigation strategies in complex environments. In In Proceedings of the IEEE International Conference on Humanoid Robots (Humanoids 2003), 2003.

[11] J.J. Kuffner, K. Nishiwaki, S. Kagami, M. Inaba, and H. Inoue. Motion planning for humanoid robots under obstacle and dynamic balance constraints. In Proceedings of the IEEE International Conference on Robotics and Automation (ICRA'01), pages 692-698, 2001.

[12] S. Kagami, J.J. Kuffne, K. Nishiwaki, Y. Kuniyoshi, K. Okada, and M. Inaba. Humanoid arm motion planning using stereo vision and rrt search. In In Proceedings IEEE/RSJ International Conference on Intelligent Robots and Systems (IROS'03), pages 2167-2172, 2003.

[13] R. Alami and T. Simeon and J.P. Laumond. A geometrical approach to planning manipulation tasks. In In Proceedings of 5th International Symposium on Robotics Research, pages 113-119, 1989.

[14] F. Gravot and R. Alami. A method for handling muliple roadmaps and its use for complex manipulation planning. In Proceedings of the 2003 IEEE International Conference on Robotics and Automation, pages 2914-2919, 2003.

[15] K. Yamane and Y. Nakamura. Synergetic CG Choreography through Constraining and Deconstraining at Will. In Proceedings of the 2002 IEEE International Conference on Robotics and Automation (ICRA '02), pages 855-862, 2002.

[16] H. Nakai, M. Inaba, and H. Inoue. Whole Body Motion Generator of Robots using RRT-Connect Planner. In Proceedings of 2003 Annual Symposium of RoboticsMechatronics (ROBOMEC '03), pages 2P2-1F-C1, 2003 (in Japanese).

[17] H. Hirukawa, F. Kanehiro, K. Kaneko, S. Kajita, K. Fujiwara, Y. Kawai, F. Tomita, S. Hirai, K. Tanie, T. Isozumi, K. Akechi, T. Kawasaki, S. Ota, K. Yokoyama, H. Honda, Y. Fukase, J. Maeda, Y. Nakamura, S. Tachi, and H. Inoue. Humanoid Robotics Platforms developed in HRP. In Proceedings of the 2003 IEEE-RAS International Conference on Humanoid Robots (Humanoids 2003), 2003. 